\title{
Rectopexia anterior laparoscópica con malla. Una segura alternativa para manejo del prolapso rectal
}

\author{
Laparoscopic anterior rectopexy with mesh. A safe \\ alternative for the management of rectal prolapse
}

\author{
Benjamín Gallo Arriaga, *,‡ B Gallo Chico, ${ }^{\S}$ R López Narváez, "I B Andrade Aguilar,§ \\ J Ibarra Rodríguez, ${ }^{\Uparrow}$ C Hidalgo Valadez, ${ }^{\ddagger}$, G Santos Vázquez ${ }^{\ddagger}$
}

\section{Resumen}

El prolapso rectal completo es la salida del recto con todas sus capas a través del ano; el prolapso externo puede ser parcial y sólo incluir la capa mucosa de la parte distal del recto con o sin los paquetes hemorroidarios internos. Esta enfermedad predomina en el género femenino (relación 10 a 1) y es más frecuente en la sexta y séptima décadas de la vida. Los pacientes con esta patología suelen tener cierto grado de incontinencia. Las técnicas quirúrgicas para su tratamiento han evolucionado con el paso del tiempo desde abordajes perineales, abordajes abdominales hasta abordajes con técnicas laparoscópicas. Se presenta el caso de paciente femenino de 45 años, fisicoculturista. Antecedente de histerectomía por miomatosis uterina, padece estreñimiento crónico. Acude a consulta por prolapso rectal reductible. Se realiza rectopexia ventral anterior con colocación de malla sin complicaciones transoperatorias. Cursó el periodo postoperatorio de manera satisfactoria, a la evaluación dos semanas después se encontraba asintomática con mejoría en el estreñimiento y sin recurrencia de prolapso. Actualmente a los nueve meses de la cirugía continúa asintomática y sin recidiva del prolapso.

Palabras clave: Prolapso rectal, rectopexia ventral, laparoscopia.
* Cirugía de Colon y Recto, Hospital Ángeles León.

‡ Departamento de Medicina y Nutrición, Universidad de Guanajuato.

$\S$ Cirugía Gastrointestinal, Hospital Ángeles León.

II Ginecología y Gineco-oncología, Hospital Ángeles León.

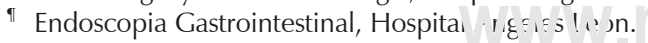

León, Guanajuato.

Correspondencia:

Dr. Benjamín Gallo Arriaga

Correo electrónico: benjasgallo@gmail.com

Aceptado: 21-11-2019.

www.medigraphic.com/actamedica

\section{Abstract}

Complete rectal prolapse is when all the layers of the rectum come out through the anus; the external prolapse can be partial and only include the mucosal layer of the distal rectum with or without internal haemorroids. This disease is predominant in female sex (10:1) and in the sixth and seventh decades of life. This patients frecuently have fecal incontinence. The surgical techniques for its treatment have evolved with the pass of the years. From perineal approches, to abdominal apporches to laparoscopic techniques. We present the case of a female patient 45 years old bodybuilder. Among her medical history she had a histerectomy due to miomatosis and chronic constipation. She has been seen in consultation for total rectal prolapse reductible. We performed an anterior laparoscopic ventral rectopexy with absorbable mesh without complications. She did well in the post operatory; in the evaluation at two weeks PO she was fine without prolapse, asymptomatic and with improvment of the constipation. At nine months after surgery the patient continues asymptomatic and without prolapse recurrence.

Keywords: Rectal prolapse, ventral rectopexy, laparoscopy.

\section{INTRODUCCIÓN}

El prolapso rectal completo es la salida del recto con todas sus capas a través del ano; el prolapso externo puede ser parcial y sólo incluir la capa mucosa de la parte distal del recto con o sin los paquetes hemorroidarios internos, o puede ser total e incluir todas las capas de recto. El prolapso rectal interno corresponde al descenso del recto sin pasar del canal anal como consecuencia de una invaginación o intususcepción. Se presenta con mayor frecuencia entre la sexta y séptima décadas de la vida y tiene una relación a favor del sexo femenino de 10:1. ${ }^{1}$ 
Cuando el prolapso rectal es completo por lo regular no es necesario realizar estudios radiológicos para su diagnóstico, sólo se requiere aplicar la maniobra de Valsalva; sin embargo, si existe la necesidad de algún estudio, hoy contamos con la cinedefecografía y con la defecografía por resonancia magnética con las cuales se puede establecer el diagnóstico. ${ }^{2}$ La cinedefecografía es un estudio radiológico que utiliza radiación ionizante, requiere la administración de contraste rectal, vaginal, vesical y en intestino delgado y es operador dependiente; la defecografía por resonancia magnética no requiere la aplicación de contraste, no conlleva radiación ionizante, es menos operador dependiente y permite una mejor definición de las estructuras y planos anatómicos. $^{3}$

Hasta $75 \%$ de los pacientes con prolapso rectal completo presentan algún grado de incontinencia, la cual por lo general es multifactorial; puede deberse al daño del esfínter anal por el prolapso mismo (dilatación continua del esfínter anal por la presencia del prolapso) o bien a lesión de los nervios pudendos por tracción de los mismos. Sin embargo, también se puede encontrar estreñimiento en estos pacientes hasta en $50 \%$ de casos, siendo éste más difícil de explicar que la incontinencia. ${ }^{4}$

El tratamiento del prolapso rectal es quirúrgico y existen numerosas técnicas quirúrgicas para la reparación del mismo, abordajes abdominales y perineales con diferentes tasas de éxito, complicaciones y morbilidad. Es generalmente aceptado que los abordajes abdominales tengan menos porcentaje de recidiva y mejores resultados funcionales. En general, los procedimientos perineales se reservan para pacientes que no son buenos candidatos para un abordaje abdominal como pacientes ancianos o con enfermedades severas asociadas, en varones jóvenes por el riesgo de disfunción sexual por lesión del plexo hipogástrico y en pacientes psiquiátricos institucionalizados debido a que estos abordajes producen poco dolor. ${ }^{5}$ El objetivo del tratamiento será restablecer la anatomía normal en el paciente y, si es posible, restablecer la función anorrectal. ${ }^{1}$

Los abordajes abdominales difieren entre sí en la extensión de la movilización rectal, el método para la fijación del recto, y en que pueden ser con o sin sigmoidectomía. ${ }^{1}$ El uso de mallas para realizar la fijación rectal se ha vuelto popular desde 1992 cuando Berman y colaboradores publicaron el primer trabajo sobre la rectopexia laparoscópica y demostraron que ya sea con sutura o con malla ambos son factibles por vía laparoscópica y tienen buenos resultados en cuanto a recidivas, estreñimiento e incontinencia. ${ }^{5}$

La rectopexia anterior es un procedimiento descrito por el Dr. D'Hoore, en el cual la disección sólo se hace en la cara anterior del recto por el tabique recto-vaginal hasta la altura del piso de la pelvis a nivel de los músculos eleva- dores del ano; se coloca una malla de aproximadamente $15 \mathrm{~cm}$ de largo por $4 \mathrm{~cm}$ de ancho que se fija con sutura absorbible a la cara anterior del recto y después se fija al promontorio sacro con suturas o tackers; pueden usarse mallas sintéticas absorbibles o malla biológicas. ${ }^{6}$

\section{CASO CLÍNICO}

Paciente femenino de 45 años quien acudió a la consulta externa por presentar un prolapso rectal total de dos meses de evolución. La paciente refirió que el prolapso era reductible manualmente y no se acompañaba de incontinencia y que se dio cuenta del prolapso al estar realizando ejercicio con pesas en exceso (fisicoculturista) durante sus sesiones de entrenamiento, pero al paso de tres semanas el recto se exteriorizaba con el mínimo esfuerzo.

Entre los antecedentes de la paciente. Quirúrgicos: histerectomía laparoscópica, apendicectomía laparoscópica, tres cesáreas. Niega antecedentes crónico degenerativos y alérgicos. Refiere estreñimiento crónico desde los 16 años, el cual responde por lo general al uso de laxantes (habitualmente laxantes irritantes con senósidos o picosulfato de sodio).

A la exploración física el prolapso rectal total era evidente, de hecho se redujo manualmente en la consulta. Se realizó colonoscopia (la cual fue normal); colon por enema en el cual no se apreció sigmoides redundante y la cinedefecografía mostró el prolapso rectal total evidente.

Se decidió entonces realizar una rectopexia ventral por laparoscopia con el uso de una malla absorbible. Escogimos este abordaje debido a la limitada disección en la pelvis, y porque tiene pocas alteraciones funcionales postoperatorias reportadas en la literatura, además se utilizó una malla bioabsorbible de ácido poliglicólico y carbonato de trimetileno debido a que con este tipo de mallas hay menos riesgo de perforación rectal o vaginal y no aumenta la posibilidad de recidiva del prolapso.

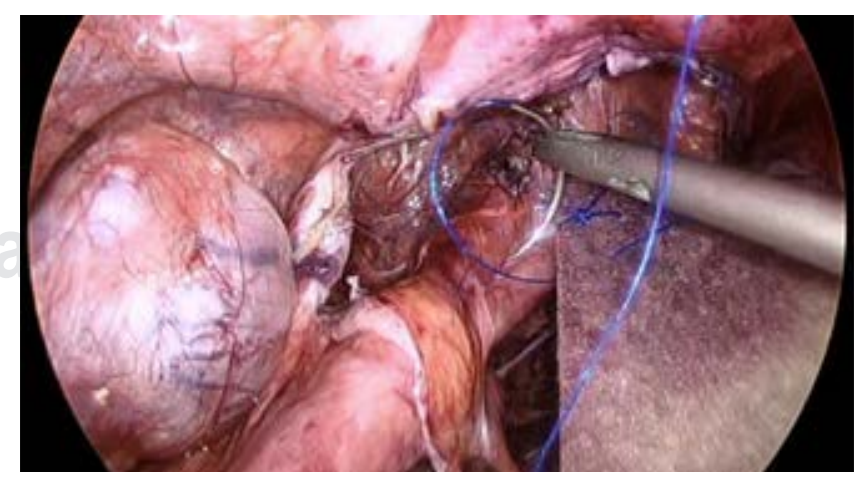

Figura 1: Puntos simples de la malla a la cara anterior del recto. 


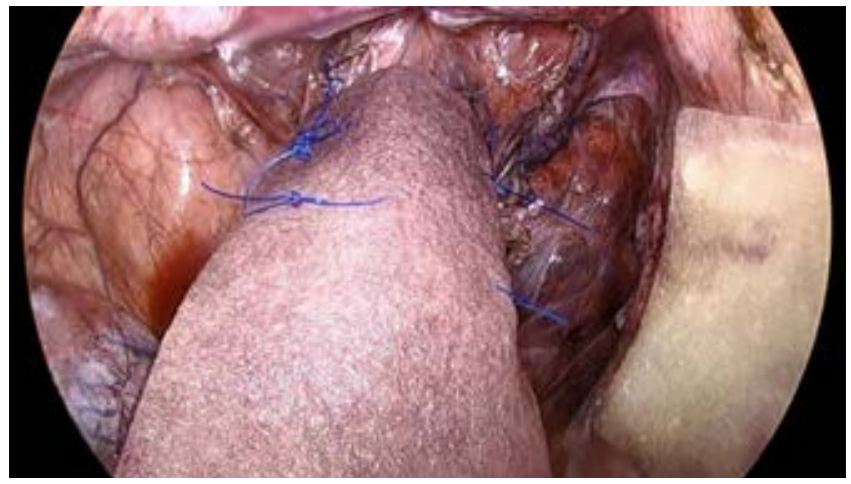

Figura 2: Tres puntos separados a cada lado de la malla con el recto.

\section{Técnica quirúrgica}

Se realizó una preparación colónica completa con polietilenglicol y antibióticos orales (preparación de Nichols-Condon) y se utilizaron antibióticos profilácticos (ertapenem 1 g IV y metronidazol 500 mg IV) en la inducción anestésica.

Con la paciente en posición de Lloyd-Davis se utilizaron cuatro trócares, uno supraumbilical $12 \mathrm{~mm}$ (óptico), otro de $12 \mathrm{~mm}$ en fosa iliaca derecha, dos de $5 \mathrm{~mm}$, uno en flanco derecho y otro en flanco izquierdo. Se inició con la apertura del peritoneo desde el promontorio sacro (identificando el periostio) del lado derecho del recto hasta el extremo izquierdo del recto abriendo el saco de Douglas mediante uso de bisturí harmónico, posteriormente se abordó el tabique recto-vaginal disecándolo hasta llegar a los músculos del piso pélvico (músculos elevadores del ano); no se realizó movilización de la cara posterior ni de los alerones laterales del recto. Una vez disecada por completo la cara anterior del recto se fijó una malla (de $12 \times 4 \mathrm{~cm}$ ) a la cara anterior del recto lo más distal posible dando puntos en la capa muscular del recto (de $3 \mathrm{~cm}$ de cada lado de la malla) con sutura monofilamento absorbible (Figuras 1 y 2). Una vez suturada la malla se tracciona el recto con ésta, fijándola al promontorio sacro con tres tackers (Figura 3). Se verifica la rectificación del recto y se sutura el peritoneo con una sutura barbada absorbible, dejando la malla extraperitoneal (Figura 4).

La paciente evolucionó satisfactoriamente, tuvo poco dolor en el postoperatorio, inició dieta normal al día siguiente de la cirugía, tuvo una evacuación normal a la mañana del segundo día y sin evidencia de prolapso durante la evacuación ni durante la exploración con maniobra de Valsalva. Se decidió su egreso hospitalario ese mismo día.

A las dos semanas la paciente se encontró asintomática, sin recidiva del prolapso y con mejoría del estreñimiento crónico, se ha mantenido sin necesidad de uso de laxantes. A los nueve meses de postoperada (operada en febrero de 2019) la paciente continúa sin recidiva, con mejoría del estreñimiento y sin ningún síntoma.

\section{DISCUSIÓN}

El tratamiento del prolapso rectal se ha modificado con el tiempo acorde a la evidencia disponible, desde cirugías amplias abdominales, más tarde perineales, hasta llegar a ser mínimamente invasivas, con excelentes resultados. ${ }^{7}$ En el caso de nuestra paciente, la elección de esta vía se debió a su edad, ausencia de comorbilidades significativas, la poca morbilidad que conlleva este procedimiento al sólo disecar el tabique recto-vaginal sin alterar la inervación rectal y el resto de su anatomía.

El tipo de abordaje quirúrgico del prolapso es aún sujeto de debate, depende sustancialmente de las comorbilidades del paciente y de las preferencias de los cirujanos. Russell y colaboradores encontraron que en los hospitales participantes del Proyecto Nacional de Mejora en Cirugía en los Estados Unidos los procedimientos perineales y abdominales se practican con igual frecuencia, pero los pacientes tratados vía abdominal tenían más complicaciones, particularmente infecciosas, en pacientes con un alto IMC o una clasificación de ASA de 4 o mayor. Debido a esto los autores recomiendan procedimientos menos invasivos como abordajes perineales o laparoscópicos, sobre todo en pacientes obesos o con otras comorbilidades. $^{8}$

En nuestra paciente se decidió la rectopexia ventral laparoscópica con malla absorbible. Se descartó el procedimiento de Frykman-Goldberg, pues la paciente no tenía sigmoides redundante; además en la rectopexia ventral con malla se limita la disección de la pelvis sólo a la cara anterior, con lo que se disminuye el riesgo de hemorragia presacra, la lesión de plexos nerviosos presacros y evita la

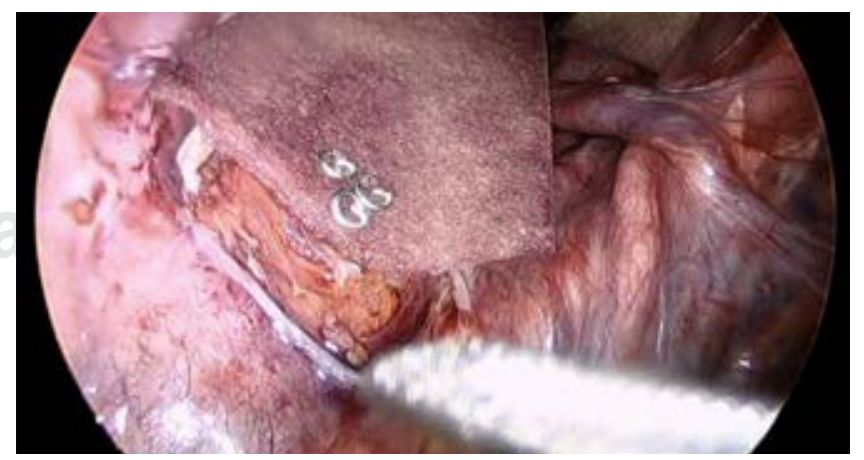

Figura 3: Fijación de la malla al promontorio del sacro con takers. 


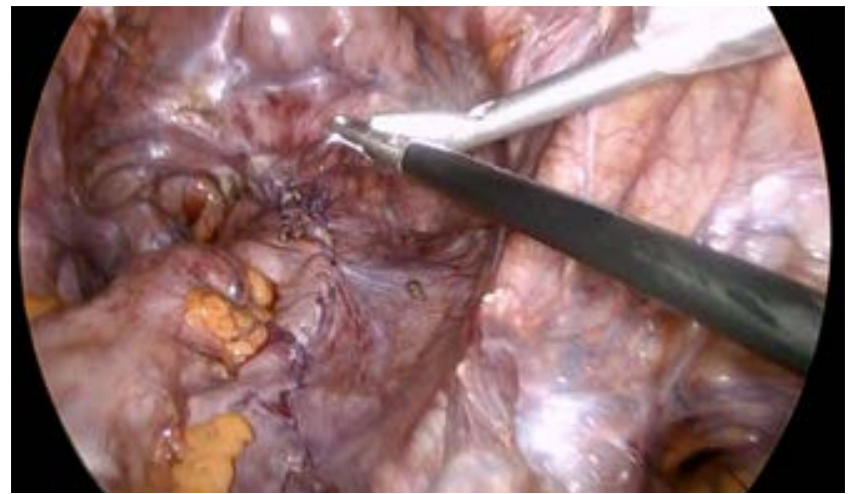

Figura 4: Una vez cerrado el peritoneo se deja la malla completamente extraperitoneal.

movilización posterior del recto, lo que favorece una menor incidencia de estreñimiento postquirúrgico. ${ }^{9}$

La vía de abordaje abdominal es la ideal en pacientes jóvenes sanos que toleran mejor este abordaje, mientras que los perineales son más aceptados en pacientes mayores o con comorbilidades asociadas. La rectopexia es la técnica preferida por muchos cirujanos y la manera de fijarla mediante sutura ofrece la misma seguridad que con el uso de mallas. ${ }^{5}$ El uso de mallas biológicas reduce las complicaciones del uso de las mismas. ${ }^{10}$

La calidad de vida de los pacientes postoperados de esta patología a través de abordajes laparoscópicos hasta hace poco ha sido evaluada de forma objetiva, ya que la mejoría anatómica puede o no correlacionar con los síntomas del paciente, siendo este último punto el más importante para la efectividad del tratamiento. En el estudio de Mäkelä-Kaikkonen y colaboradores cuando se evaluó el resultado funcional de $70-90 \%$ de los pacientes mostró mejoría en caso de incontinencia, y de $60-70 \%$ en caso de estreñimiento, así como leve mejoría en dolor pélvico y función sexual. Los síntomas que se presentaron y causaron deterioro en la calidad de vida fue la urgencia defecatoria hasta en $26 \% .^{11}$

En su estudio, Madbouly y su equipo demostraron que en pacientes ancianos la rectopexia ventral laparoscópica y el procedimiento de STARR son seguros y mejoran la función anorrectal en pacientes con prolapso rectal interno; la rectopexia ventral laparoscópica tiene mejores resultados, menos complicaciones y menos recurrencias en relación con síntomas defecatorios y el prolapso mismo. ${ }^{12}$ Otro punto a favor para recomendar este procedimiento en pacientes jóvenes.

Respecto a la seguridad de la rectopexia ventral anterior laparoscópica, se ha evaluado a través de los resultados postoperatorios. El riesgo de erosión posterior a la colocación de la malla es $<2 \%$ más frecuente en mallas sintéticas, específicamente las de poliéster; las complicaciones no asociadas con la colocación de la misma se presentaron en $11 \%$, mientras que la tasa de mortalidad a 30 días fue $0.1 \% .{ }^{10}$

En relación con la recurrencia del prolapso rectal exteriorizado posterior a la rectopexia ventral laparoscópica con malla Emile y colaboradores reportan una recidiva del prolapso rectal de $2.8 \%$ con seguimiento a dos años ${ }^{13}$ y Consten y su equipo describen una recidiva de $4.2 \%$ a los tres años en 212 pacientes operados por prolapso rectal total. ${ }^{14}$ Algunos de los factores de riesgo de recidiva son la latencia prolongada de nervios pudendos, el uso de mallas sintéticas en comparación con biológicas, edad mayor de 70 años y un estado prequirúrgico severamente incontinente, por lo que esta complicación, pese a ser infrecuente, debe tenerse presente en población de riesgo. ${ }^{15}$

En otro estudio reciente Abhijit y colaboradores evalúan 25 pacientes con prolapso rectal total operados con esta técnica y reportan sólo un paciente con recidiva del prolapso. Concluyen que este procedimiento tiene la ventaja del abordaje de mínima invasión con baja morbilidad, bajo porcentaje de recidiva y con buenos resultados funcionales respecto a la constipación y a la incontinencia fecal. ${ }^{16}$

Samarnayake y colegas en una revisión sistemática de 12 series no randomizadas de rectopexia ventral que incluyeron 728 pacientes, reportan que gracias a la baja recurrencia a mediano y largo plazo y a resultados favorables respecto a la mejoría del estreñimiento, así como de la incontinencia fecal, la rectopexia ventral por laparoscopia está emergiendo como el procedimiento de elección actual para el tratamiento del prolapso rectal total. ${ }^{17}$

\section{CONCLUSIONES}

El prolapso rectal es una patología poco frecuente asociada con factores de riesgo bien establecidos. Su identificación oportuna y la de las patologías asociadas a ésta, como incontinencia, permiten un tratamiento adecuado para resolver la enfermedad y las enfermedades asociadas, evitando así complicaciones a largo plazo.

Los abordajes abdominales tienen mejores resultados funcionales y menos recidivas que los procedimientos perineales.

El método de fijación del recto al sacro en la rectopexia mediante suturas o la colocación de mallas es igualmente eficaz.

La rectopexia ventral con malla por vía laparoscópica es un tratamiento efectivo, con bajas recurrencias reportadas en la literatura (entre 2.8 y $4.2 \%$ ) y pocas complicaciones 
derivadas de la colocación de la malla, las cuales se reducen aún más con el uso de mallas absorbibles.

\section{REFERENCIAS}

1. González CQH, Bahena-Aponte JA. Prolapso rectal. Cir Gen. 2011; 33 (Suppl: 1): 54-56.

2. Dunn K, Madoff R. Rectal prolapse. In: Wexner S, Stollman N (eds). Diseases of the colon. Taylor \& Francis; 2016.

3. O'Brien A. Defecografía por resonancia magnética. Rev Chil Radiol. 2010; 16 (1): 11-16.

4. Cannon J. Evaluation, diagnosis, and medical management of rectal prolapse. Clin Colon Rectal Surg. 2017; 30 (01): 16-21.

5. Muñoz F, del Valle E, Rodríguez M, Zorrilla J. Prolapso rectal. ¿Abordaje abdominal o perineal? Estado actual. Cir Esp. 2005; 78 (Supl 3): 50-58.

6. D'Hoore A. Rectal prolapse, intussusception, solitary rectal ulcer. In: Coloproctology. Berlin, Heidelberg: Springer Berlin Heidelberg; 2017. pp. 135-146.

7. Eftaiha S, Mellgren A. Rectal prolapse: what is the best approach for repair? In: Difficult Decisions in colorectal surgery. Springer; 2017. pp. 347-358.

8. Russel $\mathrm{M}$, Read T, Roberts $\mathrm{P}$ et al. Complications after rectal prolapse surgery: does approach matter? Dis Colon Rectum. 2012; 55 (4): 450-458.

9. Pérez Reyes M, González Poveda I, Mera Velasco S, Ruiz López M, Santoyo Santoyo J. Rectopexia ventral laparoscópica. Cir Andal. 2018; 29 (4): 557-559.
10. Evans C, Stevenson A, Sileri P et al. A multicenter collaboration to assess the safety of laparoscopic ventral rectopexy. Dis Colon Rectum. 2015; 58 (8): 799-807.

11. Kaikkonen-Makela J, Rautio T, Kairaluoma M et al. Does ventral rectopexy improve pelvic floor function in the long term? Dis Colon Rectum. 2018; 61 (2): 230-238.

12. Madbouly KM, Mohii AD. Laparoscopic ventral rectopexy versus stapled transanal rectal resection for treatment of obstructed defecation in the elderly: long-term results of a prospective randomized study. Dis Colon Rectum. 2019; 62 (1): 47-55.

13. Emile SH, Elfeki H, Shalaby M, Sakr A, Sileri P, Wexner SD. Outcome of laparoscopic ventral mesh rectopexy for full-thickness external rectal prolapse: a systematic review, meta-analysis, and metaregression analysis of the predictors for recurrence. Surg Endosc. 2019; 33 (8): 2444-2455.

14. Consten EC, van lersel JJ, Verheijen PM, Broeders IA, Wolthuis AM, D'Hoore A. Long-term outcome after laparoscopic ventral mesh rectopexy: an observational study of 919 consecutive patients. Ann Surg. 2015; 262 (5): 742-747; discussion 747-748.

15. Cherylin F, Stevenson A. Risk factors for recurrence after laparoscopic ventral rectopexy. Dis Colon Rectum. 2017; 60 (2): 178-186.

16. Chandra A, Singh P, Kumar S, Chopra N, Gupta V, Joshi P et al. Laparoscopic ventral rectopexy: A viable option in procidentia with redundant sigmoid - An Indian perspective. J Minim Access Surg. 2018; 14 (4): 304-310.

17. Bhandarkar DS. Laparoscopic rectopexy for complete rectal prolapse: mesh, no mesh or a ventral mesh? J Minim Access Surg. 2014; 10 (1): 1-3. 